\title{
Analysis of Retropubic and Laparoscopic Radical Prostatectomy for Localized Prostate Cancer
}

\author{
Lijun Yang*, Huajing Ha, Bingyi Li \\ Weinan Second Hospital, Shaanxi 714000,China
}

Corresponding author: Lijun Yang, Department of Urology, Weinan Second Hospital. Shaanxi 714000, China. E-mail:rain119@163.com

\begin{abstract}
Objective:To analysis of treatment effects and safety of retropubic approach and laparoscope approach of radical prostatectomy in localized prostate cancer. Methods: The observation group underwent radical prostatectomy by laparoscope approach,while the control group underwent radical prostatectomy by retropubic approach, after that, to analysis the operation indexes and complications of patients. Results: The intraoperative blood loss, length of stay and postoperative NRS score of observation group were less than control group, the operation time of the observation group was longer than that of the control group, with statistical significance $(P<0.05)$. The postoperative complication rate of observation group was lower than control group, with statistical significance $(P<0.05)$. Conclusion:Comparing with retropubic approach, laparoscope approach of radical prostatectomy for localized prostate cancer can improve the safety of surgical treatment and optimize surgical indicators, which is worthy of promotion.
\end{abstract}

Keywords

Localized prostate cancer; retropubic approach; laparoscope approach; radical prostatectomy; safety

\section{INTRODUCTION}

Prostate cancer is a common male malignant tumor, in different regions, the epidemiological survey of prostate cancer are different, with the development of modern clinical diagnostic techniques, the detection rate of early prostate cancer has been constantly improved. For early and mid-terms patients, the radical prostatectomy can be performed to improve symptoms and prolong the life time of patients ${ }^{[1]}$. Open operation is the main treatment for prostate cancer, but most of patients were elderly people with poor operation tolerance and there

(C) The Author(s) 2020. Open Access This article is licensed under a Creative Commons Attribution 4.0 International License (https://creativecommons.org/licenses/by/4.0/), which permits unrestricted use, sharing, adaptation, distribution and reproduction in any medium or format, for any purpose, even commercially, as long as you give appropriate credit to the original author(s) and the source, provide a link to the Creative Commons license, and indicate if changes were made. 
are many complications in some of them, which affects the quality of their lives. In recent years, the development of laparoscopic technique and its application in the treatment of different diseases have provided new ideas for treatment of prostate cancer ${ }^{[2]}$. In order to clear the therapeutic value of laparoscope approach of radical prostatectomy in localized prostate cancer. This paper combined with 76 cases of prostate cancer patients admitted to our hospital from January 2018 to December 2019, and the data of different radical operation treatment for them are as follows.

\section{MATERIALS AND METHODS}

\subsection{General data}

76 patients with localized prostate cancer were admitted to our hospital from January 2018 to December 2019. They were divided into the observation group and control group according to their different treatment intentions, and there were 38 cases in each group. The control group: patient's age 51 76, with an average age of (63.57 \pm 3.16$)$, the prostate-specific antigen (PSA) was $(6.29 \pm 3.14) \mathrm{ng} / \mathrm{mL}$. Clinical stages: Ct1 was 20 cases, $\mathrm{Ct} 2$ was 18 cases. The observation group: patient's age 50 74, with an average age of(63.55 3.09), the prostatespecific antigen(PSA) was $(6.31 \pm 3.15) \mathrm{ng} / \mathrm{mL}$. Clinical stages: $\mathrm{Ct} 1$ was 21 cases, Ct2 was 17 cases. There were little differences in general data between two groups, therefore, showing no statistical significance $(P>0.05)$.

\subsection{Inclusion criteria and exclusion criteria Inclusion criteria:}

1) Localized prostate cancer diagnosed by puncture biopsy.

2) Patients informed and signed "informed consent",[3].

3) The study was approved by Ethics Committee.

4) Imageological examination showed no systemic lesion metastasis.

5) Life expectancy predicted to be more than 2 years.

\section{Exclusion criteria:}

1) Patients with poor operation tolerance.

2) Patients with history of prostatectomy, bladder neck surgery,etc.

3) Mental disorder.

4) Contraindications to anesthesia.

5) Contraindications to laparoscopic surgery.

\subsection{Methods}

Control group:Patients underwent radical prostatectomy by retropubic approach, the surgery including a postoperative comprehensive examination. Patients should be in supine position, then, a general anesthesia was given under trachea cannula and an extraperitoneal median incision was made above the suprapubic union. After that, incise the skin and separate the tissue, dissect the pelvic node, excise the pathological tissue and send to the pathology department for diagnosis. Removing the fatty tissue of prostate to ensure the exposure of important tissues and blood vessels such as fascia endopelvina and superficial branch of deep vein. Last, according to routine methods to perform the lesion resection and set drainage tube.

Observation group: Patients underwent radical prostatectomy by laparoscope approach, the postoperative comprehensive examination was also given, the patients should be in supine position, after general anesthesia, an artificial pneumoperitoneum should be established, the Pneumoperitoneum pressure in $15 \mathrm{mmHg}$. According to the 5-hole methods, punching 5 trocars into the abdomen respectively, different trocars were distributes according to arcs, dissect the pelvic node under the laparoscope and send to the pathology department. Performing the lesion resection and whole surgery according to 7-step methods, the Pneumoperitoneum pressure reduce to $5 \mathrm{mmHg}$ after 
the operation and then continue to explore the surgery to see whether the wound is bleeding and the severity of bleeding, and perform targeted hemostasis. Finally, place the drainage tube in the left lower abdomen.

After operation, all the patients should be taken good care of and actively given analgesic, anti infection, medication according to the doctor's advice. Nursing work should be strengthened.

\subsection{Obvervational index}

1) Index of surgical treatment of patients

2) Occurrence of postoperative complications

\subsection{Evaluation criterion}

Postoperative pain was evaluated by numeric rating score(NRS), with a score of $0-10$, the higher the score is, the more severe the pain.

\subsection{Statistical method}

Using the statistical software SPSS20.0 to analysis the data, measurement data $\bar{\chi} \pm \mathrm{s}, t$ test , count data $(n, \%), \chi^{2 t}$ test, the difference was statistically significant: $P<0.05$.

\section{RESULTS}

\subsection{Comparison of Surgical Treatment Indexes of Different Patients}

The intraoperative blood loss, length of hospital stay and postoperative score of NRS of the patients in the observation group were lower than which in the control group, and the operation time in the observation group was longer than that in the control group, which was statistically significant $(P<0.05)$. The data analysis is shown in Table 1.

Table 1 Comparison of Surgical Treatment Indexes between Two $\operatorname{Groups}(\bar{\chi} \pm s, n=38)$

\begin{tabular}{ccccc}
\hline Group & Operation Time $(\mathrm{min})$ & Intraoperative blood loss $(\mathrm{mL})$ & Score of NRS(points) & Length of hospital stay(d) \\
\hline Observation & $337.88 \pm 109.36$ & $758.31 \pm 254.42$ & $2.16 \pm 0.46$ & $16.38 \pm 0.85$ \\
Control & $194.55 \pm 60.42$ & $1447.41 \pm 460.95$ & $3.72 \pm 2.25$ & $24.05 \pm 3.96$ \\
$t$ & 10.883 & 12.416 & 6.444 & 17.965 \\
$P$ & 0.000 & 0.000 & 0.000 & 0.000 \\
\hline
\end{tabular}

\subsection{Analysis of Postoperative Complications between Two Groups}

The total incidence rate of postoperative complications in the observation group was lower than that in the control group, and the difference had statistically significance $(P<0.05)$. The data was shown in Table 2 .

Table 2 Analysis of Postoperative Complications in Two Groups[n(\%)]

\begin{tabular}{cccccc}
\hline Group & Cases & Urinary leakage & Incision Infection & Erectile dysfunction & Total \\
\hline Observation & 38 & $2(5.26)$ & $3(7.89)$ & $1(2.63)$ & $6(15.79)$ \\
Control & 38 & $5(13.15)$ & $6(15.79)$ & $4(10.52)$ & $15(39.47)$ \\
$\chi^{2}$ & $/$ & $/$ & $/$ & $/$ & 5.330 \\
$P$ & $/$ & $/$ & $/$ & $/$ & 0.021 \\
\hline
\end{tabular}

\section{DISCUSSION}

A high incidence rate of prostate cancer was found among middle-aged and elderly males. Radical prostatectomy is needed for diagnosed prostate cancer, which refers that, the prostate, capsule, bilateral seminal 
vesicle, bilateral ampullary segment of vas deferens, bladder neck and lymph nodes are removed by operation. After the related lesions and periphery resected, the bladder is reconnected with the rest of the urethra to ensure the normal voiding function of the patients after operation ${ }^{[4]}$. The cause of prostate cancer is related to many issues. Where there is a history of prostate cancer in the family, the risk of prostate cancer in their offspring is tens of times higher than that of normal people. Daily diet and sexual life of patient will also affect the incidence of prostate cancer. Localized prostate cancer, also, known as organ-localized prostate cancer, refers that the tumor does not penetrate the prostate capsule, with no lymph nodes and distant metastasis. Due to the lack of effective clinical and imaging methods to accurately judge the extent of tumor invasion, especially lymph node metastasis, Tl-ZNxM, is generally regarded as organ -localized prostate cancer. Non-localized prostate cancer refers that the tumor breaks through the prostate capsule and / or has lymph node or distant metastasis, as well as the recurrence and / or metastasis of localized prostate cancer after treatment.

At present, radical prostatectomy is mainly used for the treatment of patients with localized prostate cancer, that is, the focus is in the prostate and there is no metastasis. The normal treatment of prostate cancer is mainly through the retropubic approach, that is through an incision from the lower umbilicus to the upper edge of pubis, the lesion area and adjacent tissues were excised through the incision. Retropubic radical prostatectomy is the most commonly used surgical method for prostate cancer, which there's definite surgical experience ${ }^{[5]}$. In recent years, the development of laparoscopic minimally invasive technology has provided a new surgical possibility for the treatment of prostate cancer. In the process of treatment, several small incisions are made in different parts of the abdomen, and radical prostatectomy is performed with the help of laparoscopy.

According to the results of this study, through different surgical methods for patients with localized prostate cancer, from the perspective of surgical treatment indicators, the intraoperative blood loss, postoperative NRS score and length of hospital stay in the observation group were seperately less than those in the control group, indicating that laparoscopic radical prostatectomy has a minimally invasive advantage in the treatment of prostate cancer ${ }^{[6-7]}$. The operation time of the patients in the observation group is longer, which is because of the lack of a lot of operation clinical experience, and some regional visual fields under laparoscopy are limited, while radical prostatectomy via retropubic approach is widely used. With a wealth of surgical experience and clinical medical team, the progress of the operation can be ensured. From the point of surgical safety, the incidence of postoperative complications in the observation group was $15.79 \%$, which was lower than that in the control group $(39.47 \%)$, suggesting that laparoscopic radical prostatectomy is much safer. At the same time, the postoperative hospital stay of the observation group is short and patient can be recovered in a short time. Relatively speaking, open surgery has a time of longer hospital stay and patient will be recovered slowly. Patients are more likely to have complications in the process of receiving nursing, which will affect the overall recovery effect.

It should be noted that the specimens need to be further examined after radical prostatectomy to determine whether patients need to continue other adjuvant therapy according to the results of pathological examination, so as to prolong the survival time of patients ${ }^{[8]}$. In the follow-up study, we need to constantly summarize the surgical experience of laparoscopic radical prostatectomy in the treatment of prostate cancer, master the main issues of the operation, reduce the injury caused by laparoscopy to patients, further reduce postoperative complications and make it more in line with the treatment characteristics of elderly patients.

The author believes that, during the application of laparoscopic radical prostatectomy for localized prostate cancer, attention should be paid to the following points:

1)Doing well in risk assessment of laparoscopic treatment before operation and knowing the actual situation of patients, such as focus size, patient tolerance, anesthetic risk and so on.

2)Performance during the operation should be carried out by experienced doctors as far as possible, and 
different operations need to meet with the standard requirements, and summaries should be constantly made up during the operation so as to implement the continuous optimization of treatment methods and gradually shorten the operation time.

3) Complete various preparations before surgery, such as blood routine examination, prostate function score, urination symptoms, etc., to facilitate a reasonable choice of surgery time, and to help patients improve their symptoms in time.

4) Strengthening the nursing intervention for patients after operation.

\section{CONCLUSION}

Summing up, compared with retropubic approach, laparoscopic radical prostatectomy for localized prostate cancer can improve the safety of surgical treatment and optimize part of surgical treatments, which is much valuable in application and promotion.

\section{CONFERENCE}

[1] Hao Zhang, Haibo Xia, Guochang Bao, Xiaoyu Liu. Retropubic approach in the treatment of benign prostatic hyperplasia with bladder stones[J]. Inner Mongolia Medical Journal, 2010,42(S3):39-41.

[2] Jiayin Peng, Changqing Huang, Shaoqing Gao, Zhiren Cai, Zhaoguan Li. Clinical effect of different approaches in laparoscopic radical prostatectomy and its influence on sexual function of patients[J]. China Practical Medicine, 2020,15(10):33-35.

[3] Jiaxing Li,Qi Zhang, Jianwei Xie, Bo Yin. Observation on the effect of early urinary control recovery after cystourethral anastomosis with double needle barb suture during laparoscopic radical prostatectomy[J]. Journal of Modern Oncology, 2020,28(10):1703-1706.

[4] Cong Liu, Bing Chen, Wenjie Zhou, Zhenxing Hu. The influence of open surgery and laparoscopic radical prostatectomy on the quality of life and urodynamics of patients with early prostate cancer[J].The Practical Journal of Cancer,2020,35(02):248-251.

[5] Hao Du, Guanghai Yu, Yanlong Wang, Yu Ren. Efficacy of neoadjuvant endocrine therapy combined with laparoscopic surgery in the treatment of high-risk prostate cancer and its influence on urinary control[J]. Chinese Journal of Modern Drug Application,2019,13(24):176-177.

[6] Chenhui Zhang, Haiwen Chen, Qian Li. Effect of laparoscopic intrafascial radical prostatectomy on urinary control and sexual function recovery in patients with prostate cancer of different ages[J]. The Practical Journal of Cancer,2019,34(12):2040-2043.

[7] Shijing Chen. Analysis of clinical effect of laparoscopic radical prostatectomy combined with neoadjuvant endocrine therapy on patients with stage T_3N_0M_0 prostate cancer[J]. Contemporary Medicine,2019,25(35):12-14.

[8] Ximei Yang. Application of lean nursing mode in perioperative nursing of patients with high risk prostate cancer undergoing robotic laparoscopic radical prostatectomy[J]. Psychological Monthly,2019,14(23):152. 